\title{
Laparoscopic Right Hemicolectomy and Primary Anastomosis for Tubulovillous Polyp with Preoperative Endoscopic Tattooing as A Preventive Treatment in High Risk Colorectal Cancer Patient Case Report and Review
}

\author{
Carlos T Perzabal ${ }^{1}$, Veronica Salais ${ }^{1 *}$, Andrea Avalos ${ }^{2}$ and Cesar A Lopez ${ }^{3}$ \\ ${ }^{1}$ Division of Internal Medicine Oncology, Hospital General de Ciudad Juarez, Avenida Paseo Triunfo de la Republica, Mexico \\ ${ }^{2}$ Division of Internal Medicine Oncology, Hospital General de Chihuahua "Dr. Salvador Zubirán Anchondo, Mexico \\ ${ }^{3}$ Division of General Surgery Laparoscopy, Hospital General de Ciudad Juarez, Avenida Paseo Triunfo de la Republica, Mexico
}

*Corresponding author: Veronica Salais Michaus, Division of Internal Medicine Oncology, Hospital General de Ciudad Juarez, Avenida Paseo Triunfo de la Republica, 32340 Ciudad Juarez, Chihuahua, Mexico

\begin{abstract}
Background

VA/TVAs are thought to be the advanced precursors in the "adenoma-carcinoma" pathway. Right-sided colon cancer accounts for approximately $30 \%$ of bowel cancer in women and $22 \%$ in men, Curative treatment for right-sided colonic cancer includes right hemicolectomy with or without adjuvant chemotherapy. We present a 43-year-old female, with history of a father who died from colon cancer, she has a history of high blood pressure, obesity, and epilepsy, presenting hematochezia. A colonoscopy was performed with evidence of a granular scattered lateral growth lesion in the ascending colon, which cannot be resected by mucosectomy, which is why an endoscopic biopsy and tattoo was performed. The result of histopathology with tubulovillous polyp without evidence of dysplasia.
\end{abstract}

Keywords: Tubulovillous Polyp; Colorectal Cancer; Endoscopic Tattooing; Hemicolectomy; Laparoscopic Surgery; Preventive Treatment

Abbreviations: CRC: Colorectal Cancer; VA/TVA: Tubular Adenomas and Villous/Tubulovillous Adenomas; SSA: Sessile Serrated Adenomas: TSA: Traditional Serrated Adenomas; HP: Hyperplastic Polyps

\section{Introduction}

It is well established that colorectal cancer (CRC) develops from a series of precursor epithelial polyps [1], which include conventional adenomas, incorporating tubular adenomas and villous/tubulovillous adenomas (VA/TVA) and serrated polyps, incorporating hyperplastic polyps (HP), sessile serrated adenomas (SSA) and traditional serrated adenomas (TSA). VA/TVAs are thought to be the advanced precursors in the "adenoma-carcinoma" pathway [2]. Risk factors include advancing age, male gender, highfat, low-fiber diet, tobacco use, and excess alcohol intake (more than eight drinks a week). Individuals with a family history of polyps, colorectal cancer, and intestinal polyposis carry a higher risk of developing colon polyps [3]. Right-sided colon cancer accounts for approximately $30 \%$ of bowel cancer in women and $22 \%$ in men [4] Curative treatment for right-sided colonic cancer includes right hemicolectomy with or without adjuvant chemotherapy [5]. Depending on the pattern of growth, these tumors can be villous, tubular, or tubulovillous. A polyp with more than $75 \%$ villous features, i.e., long finger-like or leaf-like projections on the surface, is called a villous adenoma, while tubular adenomas are mainly comprised of tubular glands and have less than $25 \%$ villous 
features. A tubulovillous adenoma is referred to as an adenoma with both features. Tubular adenomas are the most common type of colonic adenomas, comprising a prevalence of more than $80 \%$ [6]. Although villous adenomas are more likely to become cancerous, this reflects the fact that they generally have the largest surface area due to their villous projections. If adjusted for surface area, all types of adenomas have the same potential to become cancerous [7]. The clinical significance of polyps arises from the fact that more than $95 \%$ of colon adenocarcinoma originate from polyps. Errors in localization account for a $6.3 \%$ rate of alteration in preoperatively colonic resection [8], endoscopic localization is highly inaccurate, with a $21 \%$ rate of error endoscopic tattooing is an alternative, although different techniques are used for tattooing, it is important to be consistent in the pattern of marking and to clearly document the method in the colonoscopy report. The authors recommend that tattoo be placed in 3 separate areas around the circumference of the lumen distal to the lesion [9]. Right colectomy is the procedure recommended for tumors proximal to the proximal transverse colon. Principles of right-sided resection include abdominal exploration for distant disease, mobilization and medialization of the right colon and hepatic flexure to allow for resection and anastomosis, and high ligation of the ileocolic pedicle and right branch of the middle colic artery [10] obtaining better post-surgical results with a minimally invasive and preventive approach.

\section{Materials and Methods}

We present a 43-year-old female, with history of a father who died from colon cancer, she has a history of high blood pressure, obesity, and epilepsy, presenting hematochezia. A colonoscopy was performed with evidence of a granular scattered lateral growthlesion in the ascending colon, which cannot be resected by mucosectomy, which is why an endoscopic biopsy and tattoo was performed (Figure 1). The result of histopathology with tubulovillous polyp without evidence of dysplasia. A preoperative protocol is started based on abdominal tomography and preoperative laboratories, with no evidence of alterations.
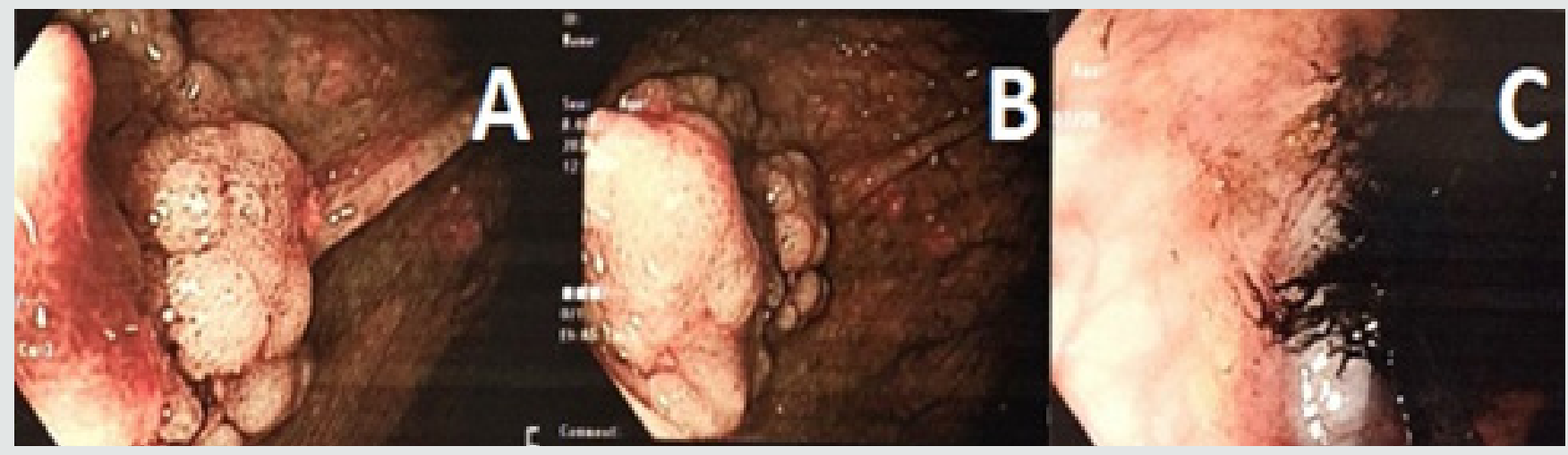

Figure 1:

A. Dispersed granular lateral growth lesion of ascending colon

B. Elevated, sessile, granular hepatic angle lesion with irregular growth and diameter of approximately $12 \mathrm{~mm}$.

C. Submucosal tattoo approximately $7 \mathrm{~cm}$ distal to the lesion

\section{Results}

Performing pneumoperitoneum at $15 \mathrm{mmHg}$, a diagnostic laparoscopy is started, the ileocecal valve is identified, an opening of the meso in the terminal ileum is performed at $10 \mathrm{~cm}$ from the valve, sectioning with a $60 \mathrm{~mm}$ endoGIA stapler, opening the right TOLD fascia, and subsequent opening of the right mesocolon with a $5 \mathrm{~mm}$ ligasure, with adequate identification of the right colic artery, the hepatic angle of the colon is released until the endoscopic tattoo is identified and the transverse colon is sectioned using an endoGIA stapler $60 \mathrm{~mm} 7 \mathrm{~cm}$ distal to the tattoo. The serous plane of the terminal ileum and transverse colon is faced laterally with 2-0 silk, a $1 \mathrm{~cm}$ opening is made in the distal portion of the ileum and colon, through which a $60 \mathrm{~mm}$ endoGIA stapler is inserted and stapling is performed, to perform side-to-side anastomosis, closure of the anastomosis with 2-0 prolene with continuous surjete, surgical piece is extracted by port in the left hypochondrium, 2 drains are left and closed by planes (Figure 2). At 24 hours after surgery, the patient had no abdominal pain, no bloating, nausea, or vomiting. The drains with little serohaematic expenditure, the patient is left fasting for 4 days and on the 5 th day an intestinal transit is carried out with a water-soluble medium without evidence of leaks (Image 4), starting a progressive liquid diet and discharging from the hospital on the 6th day without incidents or accidents (Figure 3). 

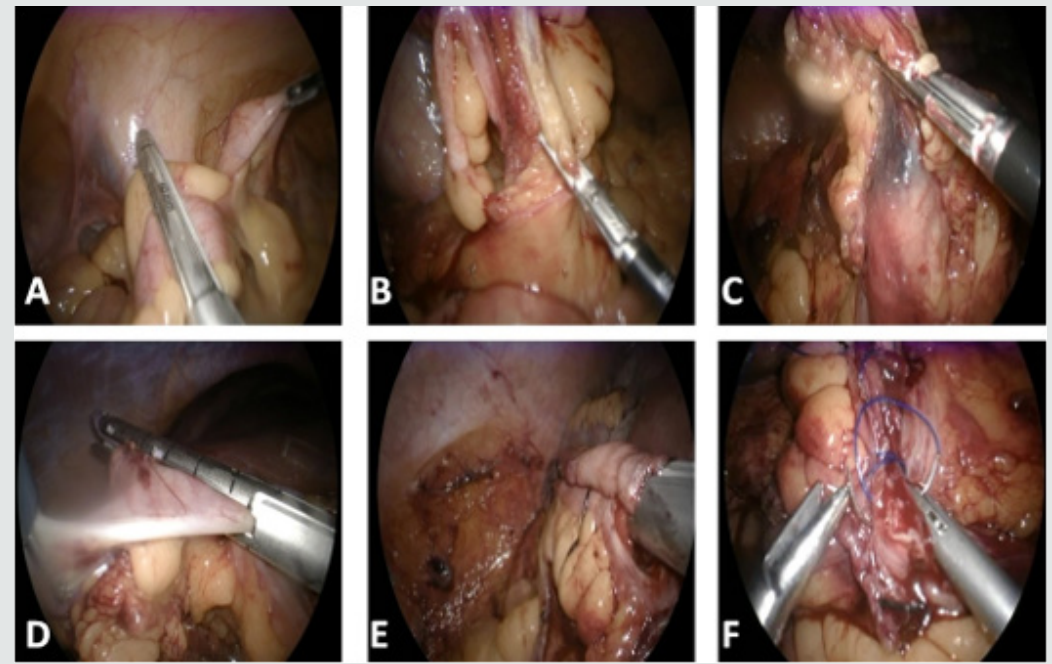

Figure 2:

A. Section of the terminal ileum at $10 \mathrm{~cm}$ from the ileocecal valve with a $60 \mathrm{~mm}$ endoGIA stapler.

B. Opening of the right mesocolon with a $5 \mathrm{~mm}$ ligasure, with identification of the right colic artery.

C. Identification of submucosal tattoo at the hepatic angle of the colon.

D. Section of the transverse colon with a $60 \mathrm{~mm}$ stapler endoGIA distal to the submucosal tattoo.

E. Lateral lateral anastomosis of the terminal ileum and transverse colon with a $60 \mathrm{~mm}$ endoGIA stapler.

F. Closure of the anastomosis with 2-0 prolene with continuous suture

Figure 3:

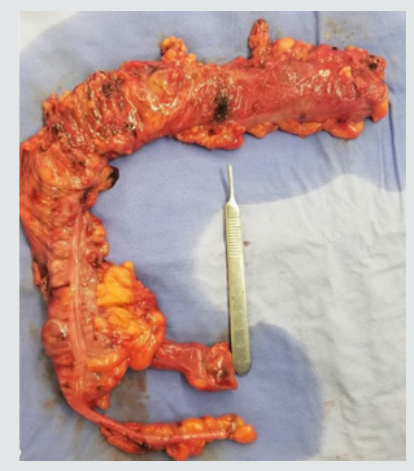

A. Right hemicolectomy surgical specimen with $10 \mathrm{~cm}$ terminal ileum and vermiform appendix. Endoscopic tattoo pointed with the scalpel.
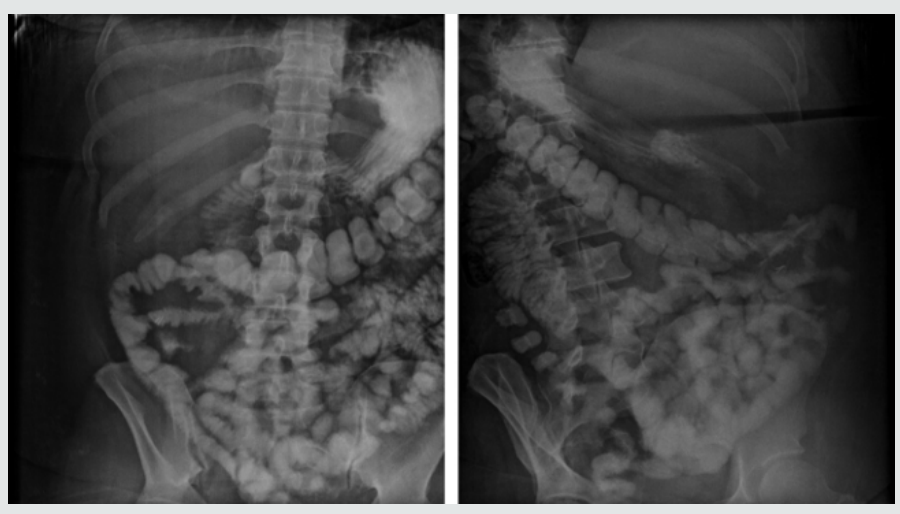

Figure 4: Anteroposterior and lateral abdominal X-ray with adequate passage of the contrast medium from the small intestine to the transverse colon, with the absence of the right hemicolon. 


\section{Discussion \& Conclusion}

A standardized approach to endoscopic tattooing will avoid confusion for the surgeon at the time of laparoscopy. This is crucial to help provide the best oncologic resection for the patient. Endoscopic tattooing is a well-known technique and helps to obtain better pre and post-surgical results with minimal invasion, however it is important to know the guidelines for the correct performance of this technique as well as take it into account to offer to patients in whom injuries are identified risk as well as concomitant hereditary factors an alternative of minimally invasive resection adequately delimiting the margins of the lesion with a faster recovery while preserving the safety of the procedure as it was presented in the case of our patient. Considering these strategies and the individualization of each patient, potential risk factors as well as clinical presentation as a therapeutic and preventive opportunity for colorectal cancer.

\section{References}

1. Cancer Research UK. Bowel Cancer Incidence Statistics. 2015.

2. Hope C, Reilly J, Lund J, Andreyev H (2020) Systematic review: the effect of right hemicolectomy for cancer on postoperative bowel function. Support Care Cancer 28(10): 4549-4559.
3. Chetty R, Hafezi-Bakhtiari S, Serra S, Colling R, Wang LM et al. (2015) Traditional serrated adenomas (TSAs) admixed with other serrated (so-called precursor) polyps and conventional adenomas: a frequent occurrence. J Clin Pathol 2015; 68: 270-273.

4. Hafezi-Bakhtiari S, Wang LM, Colling R, Serra S, Chetty R, et al. (2015) Histological overlap between colorectal villous/tubulovillous and traditional serrated adenomas. Histopathology 66: 308.

5. Myers DJ, Arora K (2020) Villous Adenoma.

6. Bujanda L, Cosme A, Gil I, Arenas-Mirave JI (2010) Malignant colorectal polyps. World J Gastroenterol 16(25): 3103-3011.

7. Yoshizawa N, Yamaguchi H, Kaminishi M (2018) Differential diagnosis of solitary gastric Peutz-Jeghers-type polyp with stomach cancer: a case report. Int J Surg Case Rep 51: 261-264.

8. Johnstone M, Moug S (2014) The accuracy of colonoscopic localisation of colorectal tu- mours: a prospective, multi-centered observational study. Scott Med J 59(2): 85-90.

9. Botoman VA, Pietro M, Thirlby RC (1994) Localization of colonic lesions with endoscopic tattoo. Dis Colon Rectum 37: 775-776.

10. Wells KO, Senagore A (2018) Minimally Invasive Colon Cancer Surgery. Surgical Oncology Clinics 27(2): 303-318.

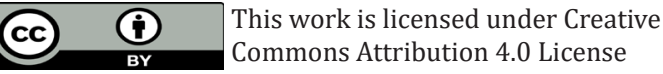

To Submit Your Article Click Here:

Submit Article
DOI: $10.32474 /$ OAJOM.2020.04.000181

Citation: Carlos T Perzabal, Veronica Salais, Andrea Avalos, Cesar A Lopez. Laparoscopic Right Hemicolectomy and Primary Anastomosis for Tubulovillous Polyp with Preoperative Endoscopic Tattooing as A Preventive Treatment in High Risk Colorectal Cancer Patient Case Report and Review. Open Acc J Oncol Med 4(2)- 2020. OAJOM.MS.ID.000181. DOI: 10.32474/OAJOM.2020.04.000181.

\section{Open Access Journal of Oncology} and Medicine

\section{Assets of Publishing with us}

- Global archiving of articles

- Immediate, unrestricted online access

- Rigorous Peer Review Process

- Authors Retain Copyrights

- Unique DOI for all articles 